\title{
Identification of Novel Cdc37 Interacting Proteins and Pathways in Human Alzheimer's Disease Brain Tissue Using Mass Spectrometry
}

Malathi Narayan ${ }^{1}$, Lisa Kirouac ${ }^{2}$, Dale Chaput ${ }^{3}$, Stanley Stevens ${ }^{3}$, Jaya Padmanabhan ${ }^{2}$ and Umesh K Jinwal $^{1 *}$

${ }^{1}$ Department of Pharmaceutical Sciences, College of Pharmacy, Byrd Alzheimer's Institute, University of South Florida Health, 4001 E Fletcher Ave, MDC36, Tampa, FL, 33613 USA

${ }^{2}$ Department of Molecular Medicine, College of Medicine, Byrd Alzheimer's Institute, University of South Florida Health, 4001 E Fletcher Ave, MDC36, Tampa, FL, 33613 USA

${ }^{3}$ Department of Cellular Biology, Microbiology and Molecular Biology, College of Art and Sciences, University of South Florida, 4001 E Fletcher Ave, MDC36, Tampa, FL, 33613 USA

"Corresponding author: Umesh K Jinwal, Department of Pharmaceutical Sciences, College of Pharmacy, Byrd Alzheimer's Institute, University of South Florida-Health, 4001 E. Fletcher Ave, MDC36, Tampa, FL, 33613 USA, Tel: +8133960673; Fax: +8139710373; E-mail: UJinwal@health.usf.edu

Received date: February 18, 2016; Accepted date: March 01, 2016; Published date: March 07, 2016

Copyright: (c) 2016 Narayan M, et al. This is an open-access article distributed under the terms of the Creative Commons Attribution License, which permits unrestricted use, distribution, and reproduction in any medium, provided the original author and source are credited.

Abstract
Alzheimer's disease (AD) is the most common form of dementia and the 6 th leading cause of death in the United
States. The major pathological hallmarks observed in AD include the formation of intracellular neurofibrillary tangles
comprised of phosphorylated forms of the microtubule associated protein tau, and the deposition of extracellular
plaques composed of amyloid beta. Cdc37 is a co-chaperone of Hsp90, which recruits client kinases to the Hsp90
complex for folding and stabilization. It has been previously shown that Cdc37 can not only bind and preserve tau,
but also stabilize kinases that can phosphorylate tau. The goal of the current study was to identify novel Cdc37-
interacting proteins in human AD tissue compared to normal tissue using an immunoprecipitation-based approach
combined with mass spectrometry. We identified 39 unique proteins that interacted with Cdc37 in AD samples only
and 7 proteins that interacted with Cdc37 in normal samples only. 39 proteins were found to bind Cdc37 in both AD
and normal tissue. Of these, 18 showed increased interaction in AD tissue, 10 showed increased interaction in
normal tissue and 11 showed equal nteraction in both samples. Ingenuity Pathway Analysis of the data indicates that
these Cdc37-interacting proteins could signal through the p70S6K, PI3K / Akt, TGFB, ErbB, NF- $k B$, calmodulin, p38
MAPK and JNK pathways. Identification of these novel proteins and pathways linked to Cdc37 may indicate its role
both as a non-kinase co-chaperone and in other pathways in the AD brain.

Keywords: Alzheimer's disease; Mass spectrometry; Proteomics; Cdc37; Ingenuity pathway analysis

\section{Abbreviations:}

AD: Alzheimer's Disease; NAD: Non Alzheimer's Disease; IP: Immunoprecipitation; MS: Mass Spectrometry; IPA: Ingenuity Pathway Analysis

\section{Introduction}

$\mathrm{AD}$ is a complex neurological disorder, which is chronic and slowly progressive with accumulation of pathology preceding symptoms by many years [1-4]. The disease may or may not have germline mutations or susceptibility alleles associated with it [5-7]. The two major pathological hallmarks of $\mathrm{AD}$ are the accumulation of neurofibrillary tangles composed of phosphorylated tau intracellularly and the deposition of plaques composed of amyloid beta peptides extracellularly $[8,9]$. The function of the microtubule-associated protein (MAP) tau is to promote the assembly and stability of microtubule networks in neurons, which is critical to normal neuronal function [10]. The binding of tau to microtubules allowing the formation of microtubule networks is regulated by phosphorylation of tau [11]. Aberrant hyperphosphorylation of tau diminishes its microtubule-binding activity and inhibits its microtubule-stabilizing function [12,13].
The cell division cycle 37 (Cdc37) genes was identified in yeast using a temperature-sensitive mutant defective in the start of the cell cycle [14]. Subsequently, it was found that Cdc37 is a kinase-specific cochaperone of heat shock protein 90 (Hsp90) [15]. Client kinases of Cdc37 include Akt, Cdk4, Cdk6, ULK1 and ULK2 [15-18]. In order to recruit kinases to the Hsp90 complex, Cdc37 functions as a scaffold binding the kinase via its $\mathrm{N}$-terminal domain and $\mathrm{Hsp} 90$ was using its central domain [19]. Upon binding to Hsp90, Cdc37 arrests the chaperone ATPase cycle allowing loading of the client kinase [19] following which a series of phosphorylation events on Hsp90 and Cdc37 lead to the dissociation of the client kinase and Hsp90 from Cdc37 [20].

Given the significance of aberrant kinase signaling in cancer and the role that Cdc37 plays in chaperoning kinases, Cdc37 has been extensively studied in cancer. A number of compounds have been developed to inhibit the interaction between Cdc37 and Hsp90, which leads to destabilization of client kinases [21-23]. The Cdc37 interactome lists over 280 proteins with a majority of them being kinases (https://www.picard.ch/downloads/Cdc37interactors.pdf). Non- kinase interactors of Cdc37 include tau and TDP-43, which are proteins implicated in AD and amyotrophic lateral sclerosis (ALS), and only recently has a function for $\mathrm{Cdc} 37$ in these neurodegenerative diseases been explored. Over-expression of Cdc37 was shown to preserve tau, while depletion of $\mathrm{Cdc} 37$ leads to clearance of tau from the cell [24]. Interestingly, in the same study it was also shown that 
Citation: Narayan M, Kirouac L, Chaput D, Stevens S, Padmanabhan J, et al. (2016) Identification of Novel Cdc37 Interacting Proteins and Pathways in Human Alzheimer's Disease Brain Tissue Using Mass Spectrometry. J Data Mining Genomics \& Proteomics 7: 193. doi: $10.4172 / 2153-0602.1000193$

Page 2 of 6

depletion of tau also leads to a decrease in kinases such as Akt and Cdk5, which are kinases that phosphorylate tau [25].

While a role for Cdc37 in tau biology is emerging, the molecular mechanism of how Cdc37 may regulate levels of tau and its kinases remains to be determined. In the current study, we undertook a mass spectrometry-based proteomic analysis of normal and $\mathrm{AD}$ brain samples to determine changes in the Cdc37 interactome in the AD brain to gain a better understanding of the role of Cdc37 in tau biology. Given that the presence of hyperphosphorylated tau is a pathological hallmark of $\mathrm{AD}$, we chose samples expressing robust levels of this form of tau. The goal of our analysis was to better understand the breadth of interactions in which Cdc37 may participate in the context of AD. Additionally, analyzing the data using Ingenuity Pathway Analysis (IPA) also allows us to identify and understand the pathways that these interactions may impinge upon further allowing us to explore non-chaperone functions of $\mathrm{Cdc} 37$. Our study has revealed the interaction of Cdc37 with a several novel proteins both in $\mathrm{AD}$ and normal brain samples. Validation of these interactions and pathways will facilitate a better understanding of not only the role of Cdc37 in $\mathrm{AD}$, but also of the Cdc37 interactome itself.

\section{Methods}

\section{Normal and AD human brain lysate}

Normal and AD tissues (Table 1) from brain cortex obtained from the University of California Irvine-Alzheimer's Disease Research Center (UCI-ADRC) were homogenized in RIPA buffer using an electric disperser homogenizer (T50 Digital Ultra-Turrax $30 \mathrm{~L}$ Disperser, IKA Works, Wilmington, NC). Following homogenization, samples were centrifuged at $15,000 \mathrm{rpm}$ for 10 minutes to pellet debris. Protein concentration was determined using the Pierce $660 \mathrm{~nm}$ Protein Assay (Thermo Scientific, Grand Island, NY).

\begin{tabular}{|l|l|l|l|l|l|}
\hline Sample \# & Age & Sex & Diagnosis & pTau & Used for MS analysis \\
\hline AD10 & 82 & Female & Late AD & + & Yes \\
\hline AD12 & 82 & Female & Late AD & + & Yes \\
\hline AD37 & 88 & Female & Late AD & + & Yes \\
\hline AD39 & 90 & Male & Late AD & - & No \\
\hline AD40 & 96 & Female & Late AD & - & No \\
\hline NAD11 & 86 & Male & Normal & - & Yes \\
\hline NAD29 & 83 & Female & Normal & - & Yes \\
\hline NAD34 & 91 & Female & Normal & - & No \\
\hline NAD40 & 91 & Female & Normal & - & No \\
\hline NAD41 & 91 & Female & Normal & - & Yes \\
\hline
\end{tabular}

Table 1: Information for normal and AD human tissue samples.

\section{Western blotting}

Normal and $\mathrm{AD}$ lysates were analyzed by Western blotting for hyperphosphorylated tau, total tau, Cdc37, Hsp90 and GAPDH as previously described [23].

\section{Immunoprecipitation of $\mathrm{Cdc} 37$-interacting proteins from normal and AD human brain lysates}

Immunoprecipitation was performed using the Pierce Crosslink Immunoprecipitation Kit (Thermo Scientific) as per manufacturer's instructions. Briefly, a monoclonal antibody to Cdc37 (Santa Cruz Biotechnology, Inc., Dallas, TX) was bound to Protein A / G Plus Agarose beads by incubated the beads with $10 \mu \mathrm{g}$ of antibody in $1 \mathrm{X}$ Coupling Buffer supplied with the kit. After incubation for 60 minutes at room temperature, the slurry was washed with Coupling Buffer three times. Following this, the bound antibody was cross linked to the beads using the DSS (disuccinimidyl suberate) cross linker in a 60 minute reaction at room temperature. $500 \mu \mathrm{g}$ of normal and AD lysates were pre-cleared using the Control Agarose Resin slurry and incubated with the Cdc37 antibody cross linked resin overnight with gentle endover-end mixing at $4^{\circ} \mathrm{C}$. Control reactions of a normal and an $\mathrm{AD}$ lysate incubated with agarose beads alone to determine the presence of non-specific interactions with Cdc37. The beads were washed with $1 \mathrm{X}$ TBS three times followed by one wash with $1 \mathrm{X}$ Conditioning Buffer. Complexes were eluted using the Elution Buffer supplied with the kit.

\section{Mass spectrometry of immunoprecipitates}

Samples were processed by filter-aided sample preparation (FASP) as described by Wisniewski and Mann [26]. Proteins were digested with Trypsin (Promega, Madison, WI) at 1:50 (w:w, enzyme:protein) overnight at $37^{\circ} \mathrm{C}$. Peptides were desalted using $\mathrm{C} 18$ columns (The Nest Group, Southborough, MA) and dried in a vacuum centrifuge. Peptide samples were resuspended in $0.1 \%$ formic acid in $\mathrm{H}_{2} \mathrm{O}$ and analyzed on a Q-Exactive Plus with a $50 \mathrm{~cm}$ UPLC column using a 90 minute gradient (2-40\% acetonitrile) on an EASY-nLC 1000 system (Thermo Fisher, Tampa, FL). Each sample was analyzed once by MS. Full MS survey scans were performed with a resolving power of 60,000, selecting the top ten most abundant ions for MS / MS fragmentation and analysis.

\section{Database searching}

Raw files were searched against the most current human (Homo sapiens) protein sequence database from UniprotKB using Maxquant (version 1.5.0.30). Search parameters included the constant modification of cysteine by carbamidomethylation and variable modification of methionine oxidation. Statistical analysis was performed using Perseus software.

\section{Pathway analysis}

Statistically significant differentially expressed proteins were used for bioinformatic analysis by Ingenuity Pathway Analysis (IPA) to determine over-represented biological functions and predicted pathway / upstream regulator activity changes.

\section{Results}

\section{Selection of AD lysates expressing hyperphosphorylated tau}

Since hyperphosphorylated tau has been predominantly linked to $\mathrm{AD}$, hence, in order to select samples expressing this pathological form of tau we screened samples (listed in Table 1) by Western blotting. We also probed for several markers including total tau, Cdc37, Hsp90 and GAPDH (Figure 1a). All samples (normal and AD) expressed total tau and Cdc37. Normal samples (labeled NAD11, NAD29 and NAD41) 
Citation: Narayan M, Kirouac L, Chaput D, Stevens S, Padmanabhan J, et al. (2016) Identification of Novel Cdc37 Interacting Proteins and Pathways in Human Alzheimer's Disease Brain Tissue Using Mass Spectrometry. J Data Mining Genomics \& Proteomics 7: 193. doi: $10.4172 / 2153-0602.1000193$

Page 3 of 6

did not express hyperphosphorylated tau. However, we found that lysates from $\mathrm{AD}$ samples ( $\mathrm{AD} 10, \mathrm{AD} 12$ and $\mathrm{AD} 37$ ) expressed the highest levels of hyperphosphorylated tau.

$\mathrm{AD}$ samples expressing robust levels of hyperphosphorylated tau were subsequently used for immunoprecipitation with an antibody to Cdc37. The antibody was cross-linked to the agarose beads used to precipitate the complexes in order to minimize the contamination of the immunoprecipitates with immunoglobulins. The immunoprecipitated samples were then digested with trypsin for analysis by mass spectrometry performed as depicted in a schematic of the experimental workflow in Figure 1b. The immunoprecipitation of Cdc37 was confirmed using a fraction of the eluted sample by Western blotting (Figure 1b). Lanes $\mathrm{C} 1$ and $\mathrm{C} 2$, in the Western blot confirming the immunoprecipitation of Cdc37, were control reactions of a normal and an AD lysate incubated with agarose beads alone to determine the presence of non-specific interactions with Cdc37.

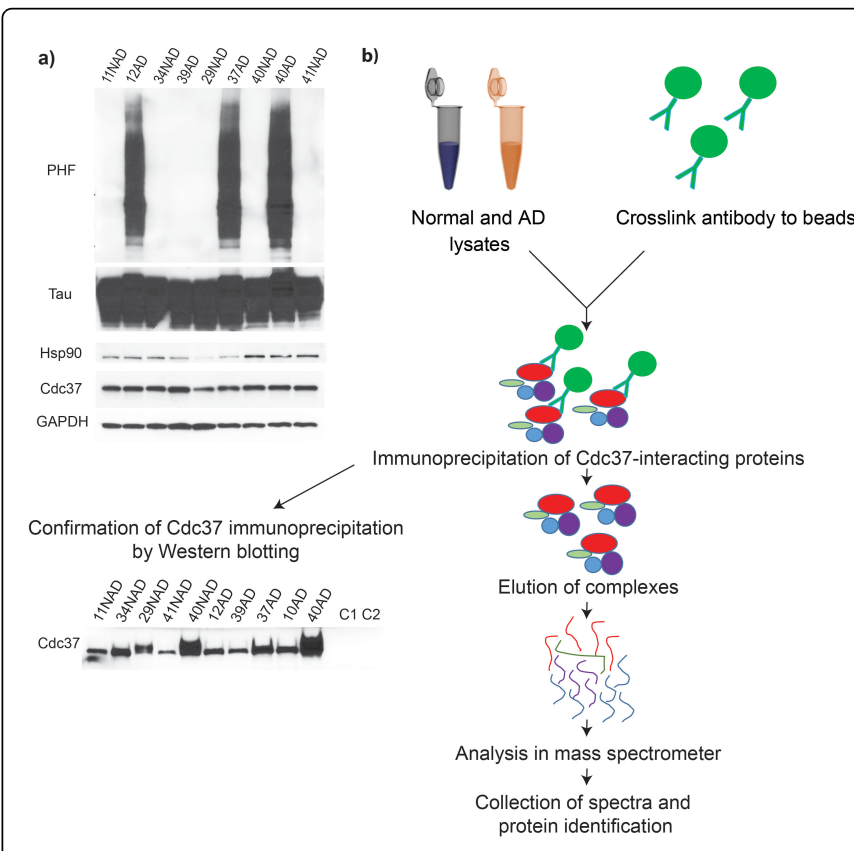

Figure 1: Western blotting analysis of human $\mathrm{AD}$ and normal samples and workflow for identification of Cdc37-interacting proteins by mass spectrometry. (a) Lysates from $\mathrm{AD}$ (AD10, AD12, AD37) and normal (NAD11, NAD29, NAD41) brain cortex samples were analyzed by Western blotting for expression of hyperphosphorylated tau, total tau, Cdc37, Hsp90 and GAPDH. (b) Schematic of the experimental workflow employed to 0identify Cdc37 interacting proteins using mass spectrometry. Immunoprecipitation of Cdc37 was confirmed by Western blotting.

\section{Analysis by mass spectrometry}

Following trypsin digestion and desalting, peptides from produced from the immunoprecipitates were analyzed by mass spectrometry leading to the identification of a total of 112 proteins. A complete list of proteins is supplied in (Supplementary Data Table 1). After removal of contaminants such as immunoglobulins from the list, 85 proteins remained. The intensities recorded for each these proteins in the $\mathrm{AD}$ and NAD samples were averaged, and the ratio of the average intensity in the AD sample to that in the NAD samples was determined. This indicated an increase, decrease or no change in the levels of these Cdc37-interacting proteins in AD samples, as indicated in Figure 2.

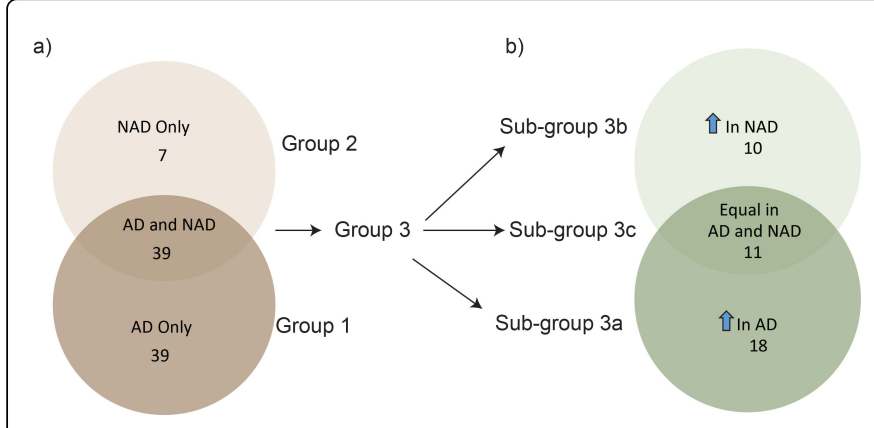

Figure 2: Venn diagram representation of Cdc37-interacting proteins. (a) Proteins identified in $\mathrm{AD}$ (Group 1) and normal samples (Group 2). Proteins found to interact with Cdc37 in both $\mathrm{AD}$ and normal samples (Group 3) are found in the overlap between Groups 1 and 2. (b) Further categorization of protein in Group 3 based on increased interaction in AD (Sub-group 3a), normal (Sub-group 3b) or equal interaction in both (Sub-group 3c).

The proteins identified in our analysis were first categorized into three groups (Supplementary Data Table 2). Group 1 included proteins that interacted with Cdc37 in AD samples only, Group 2 consisted of proteins that interacted with $\mathrm{Cdc} 37$ in normal samples only, and Group 3 contained proteins that were found to interact in both $\mathrm{AD}$ and normal samples. We found 39 unique proteins that were found to interact with $\mathrm{Cdc} 37$ in Group 1 and 7 proteins that interacted with Cdc37 only in Group 2 (Figure 2a). In Group 3, 39 Cdc37-interacting proteins were found to be common to both $\mathrm{AD}$ and normal samples. Group 3 was further separated into Sub-groups $3 \mathrm{a}$, b and c consisting of proteins that showed increased interaction in $\mathrm{AD}$ samples, normal samples or equal interaction in both and consisted of 18,10 and 11 proteins, respectively (Figure 2b). This information is also summarized in Tables 2 and 3.

\begin{tabular}{|l|l|l|l|}
\hline Group & Interactions in AD samples & Interactions in NAD samples & Interactions in both \\
\hline 1 & 39 & 0 & 0 \\
\hline 2 & 0 & 7 & 0 \\
\hline 3 & 0 & 0 & 39 \\
\hline
\end{tabular}

Table 2: Analysis of mass spectrometry data - categorization into three groups based on interactions. 
Citation: Narayan M, Kirouac L, Chaput D, Stevens S, Padmanabhan J, et al. (2016) Identification of Novel Cdc37 Interacting Proteins and Pathways in Human Alzheimer's Disease Brain Tissue Using Mass Spectrometry. J Data Mining Genomics \& Proteomics 7: 193. doi: $10.4172 / 2153-0602.1000193$

Page 4 of 6

\begin{tabular}{|l|l|l|l|}
\hline \multirow{2}{*}{ Group } & Increased interaction in AD samples & Increased interaction in NAD samples & \multirow{2}{*}{ Equal interaction in AD and NAD samples } \\
\hline $3 \mathrm{a}$ & 18 & 0 & 0 \\
\hline $3 \mathrm{~b}$ & 0 & 10 & 0 \\
\hline $3 \mathrm{c}$ & 0 & 0 & 18 \\
\hline
\end{tabular}

Table 3: Analysis of mass spectrometry data - further categorization of Group 3 based on increased interactions.

\section{Data analysis}

Analysis of proteomic data by IPA allows for data mining of pathway information related to the identified proteins and for construction of network maps that may provide information on where these pathways may intersect. Proteins in Groups 1, 2 and 3 were further analyzed using IPA to better understand the pathways and networks in which these proteins may be components.
Group 1 proteins were found to be involved in signaling via the p70S6K and the PI3K / Akt pathways (Figure 3a), while Group 2 proteins appeared to signal via TGFß and the ErbB pathways (Figure $3 b)$. Proteins in Group 3 were found to participate in the Akt, NF- $k B$, calmodulin, p38 MAPK and JNK pathways (Figures 4a - 4c).
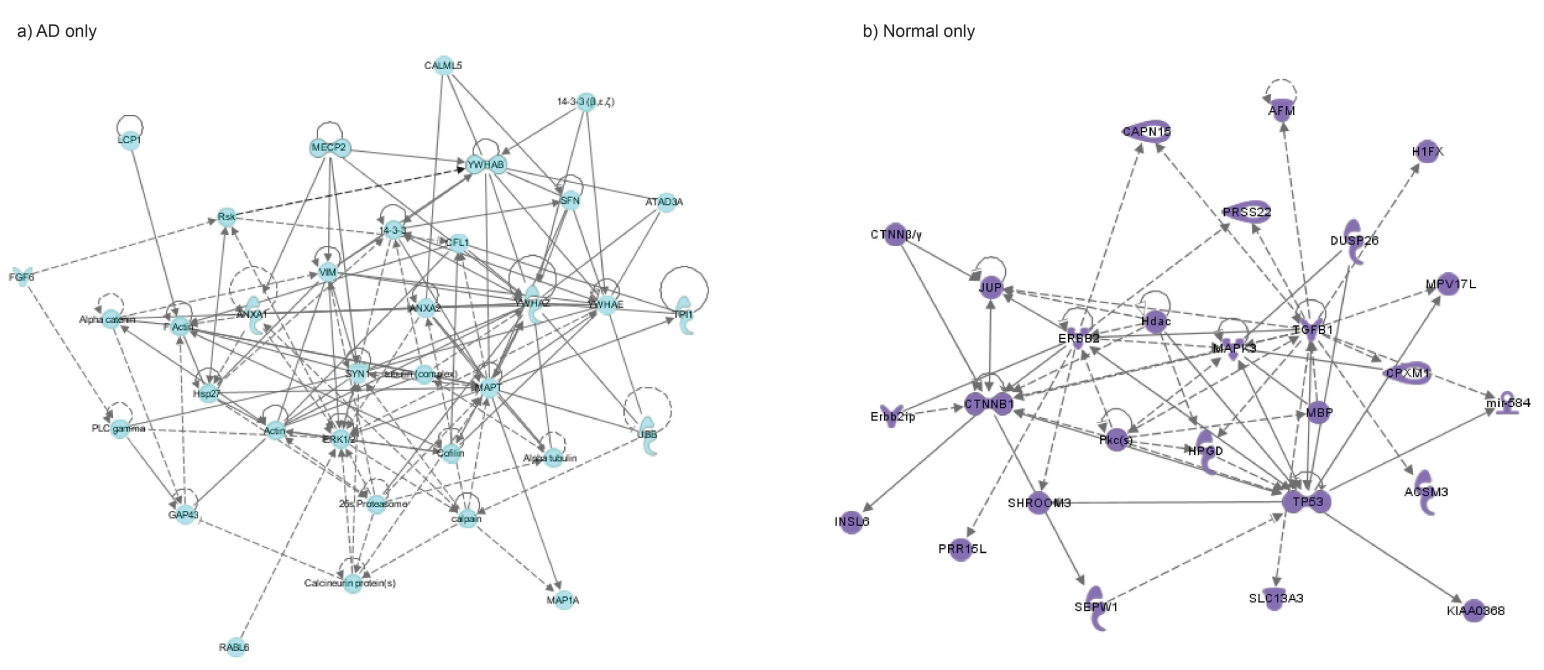

Figure 3: IPA analysis of Cdc37-interacting proteins in AD and normal samples. (3a) Pathway analysis of 39 proteins found to interact with Cdc37 only in AD samples (Group 1). (3b) Pathway analysis of 7 proteins found to interact with Cdc37 only in normal samples (Group 2). All pathway images were created using the Path Designer tool in IPA. 
Citation: Narayan M, Kirouac L, Chaput D, Stevens S, Padmanabhan J, et al. (2016) Identification of Novel Cdc37 Interacting Proteins and Pathways in Human Alzheimer's Disease Brain Tissue Using Mass Spectrometry. J Data Mining Genomics \& Proteomics 7: 193. doi: 10.4172/2153-0602.1000193

Page 5 of 6

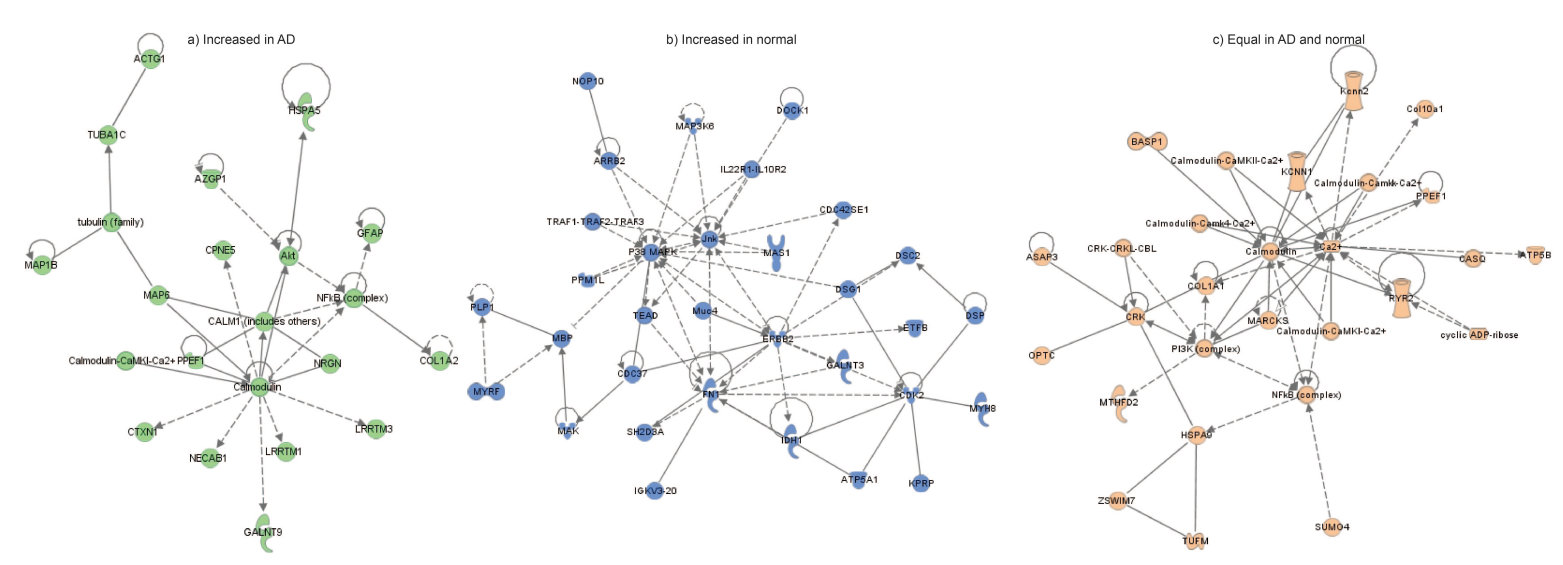

Figure 4: IPA analysis of Cdc37-interacting proteins common to both $\mathrm{AD}$ and normal samples. In Group 3, 39 Cdc37-interacting proteins were found to be common to both $\mathrm{AD}$ and normal samples. Group 3 was further separated into sub-groups consisting of (4a) proteins that showed increased interaction in AD samples, (4b) normal samples or (4c) equal interaction in both. All pathway images were created using the Path Designer tool in IPA.

\section{Discussion}

$\mathrm{AD}$ affects approximately 47 million people worldwide, a number which is expected to triple in the next 40 years [27]. While considerable headway has been made in understanding the disease, its progression and detection, there is no cure for $\mathrm{AD}$. Intracellular neurofibrillary tangles composed of hyperphosphorylated tau and extracellular plaques containing $A ß$ peptides are pathological hallmarks of the disease. However, complete understanding of the pathways regulating the formation of these species are poorly understood. Tau and kinases that phosphorylate it have previously been shown to interact with and be regulated by Cdc37, a cochaperone of Hsp90 [25]. The molecular mechanisms involved in this regulation are not fully understood. We hypothesized that identification of the components of the Cdc37 interactome in AD brain tissue would lead a better understanding of $\mathrm{Cdc} 37$ function in tau biology. In the current study, we performed mass spectrometrybased proteomic analysis of complexes obtained by immunoprecipitation of $\mathrm{Cdc} 37$ from normal and AD brain samples, and have identified a number of novel members of the Cdc37 interactome.

In the current study, we chose to immunoprecipitate Cdc37 containing complexes using a Cdc37 antibody that was chemically cross-linked to Protein G beads to minimize contamination of the eluted complexes with immunoglobulin, which was advantageous downstream during mass spectrometry analysis.

The proteins identified by mass spectrometry were analyzed by IPA, which indicates that these Cdc37-interacting proteins may be involved in several diverse pathways in cells including the PI3K / Akt, NF-B, JNK and TGF $\beta$ signaling. Some of these pathways have previously been investigated in relation to AD. Signaling through the PI3K / Akt pathway occurs in response to neurotrophic factors promoting cell survival [28]. This pathway has been found to be dysregulated in $\mathrm{AD}$ and correlates with the severity of the disease [29]. The PI3K / Akt pathway is also upstream of mTOR (mammalian target of rapamycin), which is a regulator of autophagy [30]. The disruption of PI3K / Akt / mTOR pathways is significant as it results in disruption of autophagy and abnormal accumulation of proteins leading to cell death. The JNK (c-Jun N-terminal kinase) pathway is involved in stress signaling pathways that control gene expression, neuronal plasticity and regeneration and cell death. Increased levels of phosphorylated JNK have been detected in post-mortem $\mathrm{AD}$ brain tissue [31]. Additionally, activated JNK3, which is mainly localized in neurons, is found in brain tissue and cerebrospinal fluid from $\mathrm{AD}$ patients and its presence is correlated with cognitive decline [32]. In mouse models of AD, it has been found that JNK activation is associated with an increase in senile plaques and neurofibrillary tangles [33]. Calmodulin signaling is implicated in $\mathrm{AD}$ progression as part of a 'stress-response' kinase pathway [34]. Similarly, reduction in TGFß signaling has been associated with increased deposition of $A ß$ and neurodegeneration in a mouse model [35]. The proteins identified in our study and these pathways have not previously been described in relation to Cdc37; however, further analysis and validation of these interactions may uncover a role for $\mathrm{Cdc} 37$ in these pathways.

With the identification of these novel Cdc37 interactors several questions remain to be answered. We did not identify any Cdc37interacting kinases in our study; however, it should be noted that the complexes eluted in our immunoprecipitation experiments represent a snapshot of the interactions occurring in these samples. Another factor that may contribute to the identification, or lack thereof, of certain proteins could be attributed to the abundance and level of expression of these proteins $\mathrm{AD}$ and normal tissue. Furthermore, the use of human tissue samples introduces an additional level of variability from sample to sample in terms of gender and patient bias. All of our AD samples and all but one of our normal samples that were used for mass spectrometry analysis were from female patients. Studies with a larger cohort comprising of equal numbers of samples from male and female patients and further categorized based on metrics such as ethnicity may help in strengthening these findings.

We also note that cortical tissue, composed of both neurons and glia, was used in this study. This requires that we consider the exact cellular location of these interactions. It is possible that these interactions may be occurring in neurons, glia or both. Future studies with a larger cohort of samples will give us the opportunity to address 
Citation: Narayan M, Kirouac L, Chaput D, Stevens S, Padmanabhan J, et al. (2016) Identification of Novel Cdc37 Interacting Proteins and Pathways in Human Alzheimer's Disease Brain Tissue Using Mass Spectrometry. J Data Mining Genomics \& Proteomics 7: 193. doi: $10.4172 / 2153-0602.1000193$

Page 6 of 6

these questions while also getting a better understanding of the breadth of pathways that Cdc37 may participate in. The current study provides us with novel candidate proteins that may participate in the Cdc37 interactome. Further studies will focus on understanding the significance of these novel Cdc37-interacting proteins and pathways in $\mathrm{AD}$.

\section{Acknowledgement}

This work was supported by the Byrd Small Grants Program. This work has also been supported in part by the Florida Center of Excellence for Drug Discovery and Innovation at the University of South Florida. Human tissues were obtained from the UCI-ADRC.

\section{References}

1. Galasko D (2015) Expanding the Repertoire of Biomarkers for Alzheimer's Disease: Targeted and Non-targeted Approaches. Front Neurol 6: 256.

2. Braak H, Braak E (1997) Diagnostic criteria for neuropathologic assessment of Alzheimer's disease. Neurobiol Aging 18: S85-S88.

3. Braak H, Braak E (1997) Staging of Alzheimer-related cortical destruction. Int Psychogeriatr 9: 257-261.

4. Villemagne VL, Fodero-Tavoletti MT, Masters CL, Rowe CC (2015) Tau imaging: early progress and future directions. Lancet Neurol 14: 114-124.

5. Rossor MN, Newman S, Frackowiak RS, Lantos P, Kennedy AM (1993) Alzheimer's disease families with amyloid precursor protein mutations. Ann N Y Acad Sci 695: 198-202.

6. Castellani RJ, Zhu X, Lee HG, Smith MA, Perry G (2009) Molecular pathogenesis of Alzheimer's disease: reductionist versus expansionist approaches. Int J Mol Sci 10: 1386-1406.

7. Raskin J, Cummings J, Hardy J, Schuh K, Dean RA (2015) Neurobiology of Alzheimer's Disease: Integrated Molecular, Physiological, Anatomical, Biomarker, and Cognitive Dimensions. Curr Alzheimer Res 12: 712-722.

8. Iqbal K, Alonso Adel C, Chen S (2005) Tau pathology in Alzheimer disease and other tauopathies. Biochim Biophys Acta 1739: 198-210.

9. Zhang H, Ma Q, Zhang YW, Xu H (2012) Proteolytic processing of Alzheimer's beta-amyloid precursor protein. J Neurochem 120: 9-21.

10. Wang JZ, Liu F (2008) Microtubule-associated protein tau in development, degeneration and protection of neurons. Prog Neurobiol 85: 148-175.

11. Kopke E, Tung YC, Shaikh S, Alonso AC, Iqbal K, et al. (1993) Microtubule-associated protein tau. Abnormal phosphorylation of a nonpaired helical filament pool in Alzheimer disease. J Biol Chem 268: 24374-24384.

12. Lindwall G, Cole RD (1984) Phosphorylation affects the ability of tau protein to promote microtubule assembly. J Biol Chem 259: 5301-5305.

13. Alonso AC, Zaidi T, Grundke-Iqbal I, Iqbal K (1994) Role of abnormally phosphorylated tau in the breakdown of microtubules in Alzheimer disease. Proc Natl Acad Sci USA 91: 5562-5566.

14. Reed SI (1994) The selection of Scerevisiae mutants defective in the start event of cell division. Genetics 95: 561-577.

15. Stepanova L, Leng X, Parker SB, Harper JW (1996) Mammalian p50Cdc37 is a protein kinase-targeting subunit of Hsp90 that binds and stabilizes Cdk4. Genes Dev 10: 1491-1502.
16. Basso AD, Solit DB, Chiosis G, Giri B, Tsichlis P, et al. (2002) Akt forms an intracellular complex with heat shock protein 90 (Hsp90) and Cdc37 and is destabilized by inhibitors of Hsp90 function. J Biol Chem 277: 39858-39866.

17. Lamphere L, Fiore F, Xu X (1997) Interaction between Cdc37 and Cdk4 in human cells. Oncogene 14: 1999-2004.

18. Behrends C, Sowa ME, Gygi SP, Harper JW (2010) Network organization of the human autophagy system. Nature 466: 68-76.

19. Roe SM, Ali MM, Meyer P (2004) The Mechanism of Hsp90 regulation by the protein kinase-specific cochaperone p50 (cdc37). Cell 116: 87-98.

20. Xu W, Mollapour M, Prodromou C (2012) Dynamic tyrosine phosphorylation modulates cycling of the HSP90-P50(CDC37)-AHA1 chaperone machine. Mol Cell 47: 434-443.

21. Miyata Y (2005) Hsp90 inhibitor geldanamycin and its derivatives as novel cancer chemotherapeutic agents. Curr Pharm Des 11: 1131-1138.

22. Zhang T, Hamza A, Cao X (2008) A novel Hsp90 inhibitor to disrupt Hsp90/Cdc37 complex against pancreatic cancer cells. Mol Cancer Ther 7: $162-170$.

23. Yu Y, Hamza A, Zhang $\mathrm{T}$ (2010) Withaferin A targets heat shock protein 90 in pancreatic cancer cells. Biochem Pharmacol 79: 542-551.

24. Chakrabortee S, Liu Y, Zhang L (2012) Macromolecular and smallmolecule modulation of intracellular Abeta42 aggregation and associated toxicity. Biochem J 442: 507-515.

25. Jinwal UK, Trotter JH, Abisambra JF (2011) The Hsp90 kinase cochaperone Cdc37 regulates tau stability and phosphorylation dynamics. J Biol Chem 286: 16976-16983.

26. Wisniewski JR, Zougman A, Nagaraj N, Mann M (2009) Universal sample preparation method for proteome analysis. Nat Methods 6: 359-362.

27. Wray S, Fox NC (2015) Stem cell therapy for Alzheimer's disease: hope or hype? Lancet Neurol.

28. Franke TF (2008) PI3K / Akt: getting it right matters. Oncogene 27: 6473-6488.

29. Griffin RJ, Moloney A, Kelliher M (2005) Activation of Akt / PKB, increased phosphorylation of Akt substrates and loss and altered distribution of Akt and PTEN are features of Alzheimer's disease pathology. J Neurochem 93: 105-117.

30. Manning BD, Cantley LC (2007) AKT / PKB signaling: navigating downstream. Cell 129: 1261-1274.

31. Zhu X, Raina AK, Rottkamp CA (2001) Activation and redistribution of c-jun N-terminal kinase / stress activated protein kinase in degenerating neurons in Alzheimer's disease. J Neurochem 76: 435-441.

32. Gourmaud S, Paquet C, Dumurgier J (2015) Increased levels of cerebrospinal fluid JNK3 associated with amyloid pathology: links to cognitive decline. J Psychiatry Neurosci 40: 151-161.

33. Savage MJ, Lin YG, Ciallella JR, Flood DG, Scott RW (2002) Activation of c-Jun N-terminal kinase and p38 in an Alzheimer's disease model is associated with amyloid deposition. J Neurosci 22: 3376-3385.

34. Coello GM, Polleux F (2014) Involvement of 'stress - response' kinase pathways in Alzheimer's disease progression. Curr Opin Neurobiol 27: 110-117.

35. Tesseur I, Zou K, Esposito L (2006) Deficiency in neuronal TGF-beta signaling promotes neurodegeneration and Alzheimer's pathology. J Clin Invest 116: 3060-3069. 